\title{
Desktop Virtualization Technologies and Implementation
}

\author{
Pranit Patil $^{1}$, Shakti Shekar ${ }^{2}$ \\ I' Mumbai, India) \\ ${ }^{2}$ (Mumbai, India)
}

\begin{abstract}
Desktop virtualization is new desktop delivery method in which desktop operating system instance customized, build and runs in data centers and users can access application or the whole desktop by using their stateless 'thin clients' devices. This method promises significant benefits in terms of data security, total cost of ownership and manageability of large amount of operating systems instances running in corporate intranetworks. In this paper we will analyze and compare various methods possible and used while implementing desktop virtualization and present which desktop virtualization method can be suitable with various given environments.
\end{abstract}

Keywords - cloud computing, desktop virtualization, virtualization

\section{INTRODUCTION}

Virtualization is relatively generalized term, which refers to the operation and management of computing elements on the virtual platform and as a resource solution in order to simplify the management and optimize resources. Desktop virtualization has varied definitions with the context in which it is implemented. Terminal Services been handy as it is many to one solution in which many users share same remote environment. However terminal services have had its share of problems, which have included issues with printing and application compatibility. Also individual user doesn't have full admin rights on their session. The virtual desktop interface concept sidesteps some of these problems.

Desktop virtualization also referred as virtual desktop interface. Desktop virtualization can be defined as virtualization of computer desktop in order to achieve security and flexibility. This paper presents in depth introduction to the desktop virtualization and various method involving while implementation of desktop virtualization and the suitable desktop virtualization methods for given execution environment.

\section{CURRENT STATUS OF DESKTOP VIRTUALIZATION}

The desktop virtualization products like Virtualbox, VMware Workstation which are commonly used by users does not completely realize desktop virtualization [1]. In other words, this software runs the virtual machine instance on the local or remote computer which is possible by providing and emulates the set of hardware so that it can be transferred to other machine by means of removable media or network. However the real desktop virtualization enables user to have their own desktop where she can work and save/modify data without conflicting to possible other users, which can be running on top of separate or shared operating system instance and that can be accessed via any dumb device which is having network connectivity.

There are few things one should keep in mind while deploying and provisioning of virtualized desktop. Before desktop virtualization computers were self contained. The traditional computer didn't share everyone was separate. Every PC was a self contained unit. Everything separate operating systems, applications and peripheral programs. Desktop virtualization operating costs lower due to resources being shared on a needed basis. Information is maintained and backed up in the data center[2].

The implementation of desktop virtualization can follow steps given below.

In first step, system administrator or the cloud vendor gathers information of what type of virtualization and underlying operating systems users are going to use and number of users which are going to use that particular desktop virtualized instance. It is important because it decides what type of cloud implantation the whole setup will need. 


\section{Pranit Patil, Shakti Shekar/ IOSR Journal of Engineering (IOSRJEN) \\ www.iosrjen.org \\ ISSN : 2250-3021}

Vol. 2 Issue 2, Feb.2012, pp.310-314

In second step, this step will consist of implantation of virtualization technology. It consists of isolation of individual operating system by providing emulated set of hardware devices to it. It allows the virtual desktop computer or that virtualized instance in particular to freely migrate and reuse or copy so that it can be scaled up and used on demand whenever there is need, without starting it from scratch again.

In third step, it consists of analyze and implementation of network and centralized management for better control on the number of running instances of desktop virtualization. Implementation of proper networking allows user to seamlessly access her desktop across provided network. Centralized management allows ease of control on various running virtualized instances on one or more machine within network.

In fourth step, it consists of managing availability, scalability and security. When the isolation and networking of the desktop virtualized instance are deployed, each user should get her desktop without hitch and it should support scalability to provide customized desktop on demand. Availability of virtualized desktop can be achieved by redundancy and storing that instances on network storage where it can be accessed easily. The current desktop virtualization product and software are only follows first two steps and pays little or no concentration on remaining two steps. The last two steps are currently in attention of cloud computing research as the current desktop virtualization has wide scope if it is provided with cloud at its back.

\section{IMPLEMENTATION OF DESKTOP VIRTULIZATION}

Desktop virtualization generally consists of following five modules. Those modules can be integrated in one or more computing nodes depending upon the analysis done in first step discussed in $\mathrm{I}^{\mathrm{st}}$ part. Those modules consist of Virtual machines on which desktop instance will run, Image repository which holds image data, Management Server which manages the whole infrastructure and users and Management Console, finally End User and their connectivity to the network.

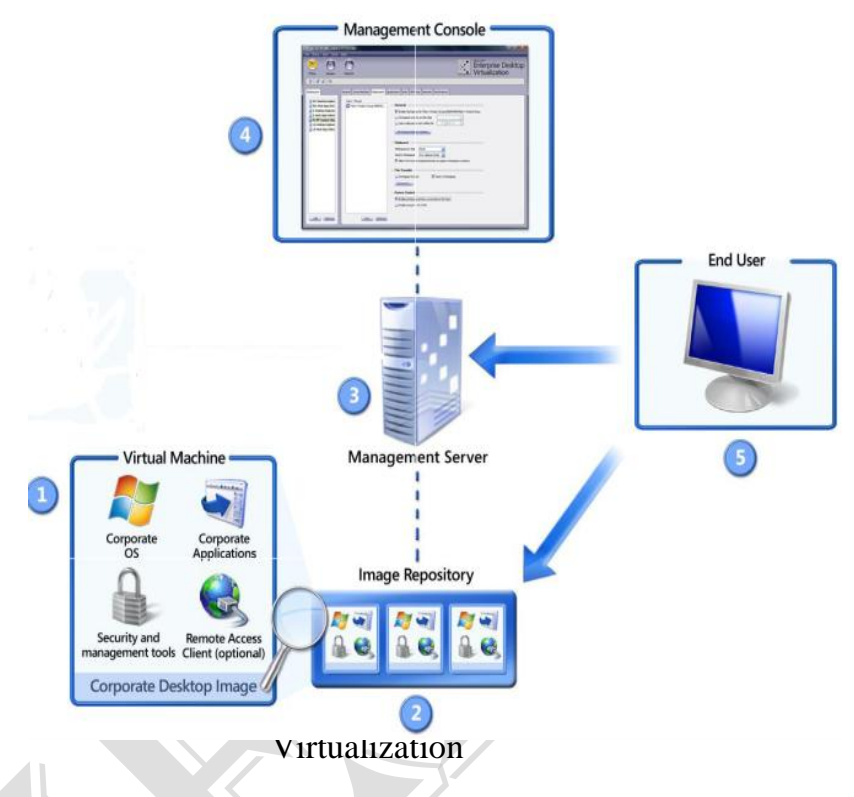

Fig. 1 shows the structure in which desktop vitalization can be implemented. It shows virtual machines, management console, image repository, management server achieves desktop virtualization by interacting with each other.

\section{FUNCTIONS OF EACH MODULES OF DESKTOP VIRTUALIZATION SYSTEM}

The first module is Virtual Machine: Virtual machine consists of physical server and the host operating system which runs the virtualized instance of operating systems which can be provided to end users. It also consists of security and management tools customize and remote access server to facilitate remote control on that virtual machines.

The second module is Image repository: It consists of central management and storage system for images of desktop instances. Image repository allows facilitating various important services like data backup, image replication, snapshot storage of images and recovery storage. This modules is generally implemented on dedicated storage boxed like fiber SAN or gigabit NAS.

The third module is Management server: Management server consists of central management mechanisms with the help of management software. This module allows 


\section{Pranit Patil, Shakti Shekar/ IOSR Journal of Engineering (IOSRJEN) \\ www.iosrjen.org \\ ISSN : 2250-3021 \\ Vol. 2 Issue 2, Feb.2012, pp.310-314}

central and easy efficient control on all other modules. It controls Virtual machine modules and Image Repository and provides end user the 'Management Console[3]'

The fourth module is Management Console: Management console is provides by management server for end user. End users who want virtualized remote desktop can log in and make request for the specific or operating system desktop assigned to them. Management console is ultimate place where user can manage and configure and perform roles assigned to them by management server [4].

The fifth and final module in End User: End user module not only consists of person and end node which accesses the virtualized desktop but also the network which connect that node to the management server and image store. End users can place their request on management server and can get virtualized image or desktop from images store and virtual machines.

\section{DESKTOP VIRTULIZATION TECHNOLOGIES}

Virtualization technology is used to solve separation of hardware and operating system. Desktop virtualization is the concept of isolation a logical operating system (OS) instance from client that we used to access it. [4] Virtualization also allows run multiple instances of desktop virtualized instances on single physical server. So considering these benefits it is getting lot of attention and rapid development and support from IT industry. Apart from the generic model described in fig. 1, desktop virtualization can be implemented or operated in specifically following modes [6].

1) Hosted virtual desktops: Hosted virtual desktops result from desktop virtualization services provided through an outsourced, hosted subscription model. Hosted virtual desktop services generally include a managed desktop client operating system configuration. Transferring information technology infrastructure to an outsourced model can shift accounting for the associated costs from capital expenses to operating expenses. According to a report by Gartner, hosted services accounted for more than 500, 00 desktop units as of March 2009 but will grow to 49 million desktop units by 2013 and may make up $40 \%$ of the worldwide 'professional PC market' by revenue [7]

2) Centralized virtual desktops: With this model, all virtualized desktop instances are hosted on one or more centralized servers. All the data associated with it are stored on direct or networked storage devices attached to those centralized servers. In this mode there can be one to one mapping of desktop to user known as static mode or could be master image which is accessed by every each user and their generated data can be stored in other location, this is also known as dynamic mapping.

3) Remote Synchronized virtual desktops: In this mode, first image is copied to a local system, where it may run without requiring a network connection. Images are 'checked out' for period of time and typically need to be refreshed periodically. Here the local machine runs host OS along with hypervisor which in turns runs virtualized instance of desktop OS with help of the synchronized image.

\section{DESKTOP VIRTUALIZATION REQUIREMENTS}

One of the primary concerns for companies implementing desktop virtualization is to understand infrastructure needed to deploy it. It is varied according to the number of virtualized instances of desktop needed, scalability and modes of deployment. Basic requirements can be grouped into server, network and storage.

1) Server requirements: The system CPU and memory requirements are based on requirements. CPU's can be shared as many hypervisors supports and provides virtual CPU independent of physical processors of system. Memory is not easy to share and it is not recommended so system physical memory should be good enough to smoothly run each virtualized desktop instance. Typically for smoothly running, each instant should have at least 512MB dedicated memory.

2) Network requirements: All the network connection between servers and clients, internal server to server and storage devices should be properly configured and well enough to handle peak network traffic and should be scalable. Regardless of the apparent hassle, the network 


\section{Pranit Patil, Shakti Shekar/ IOSR Journal of Engineering (IOSRJEN) \\ www.iosrjen.org \\ ISSN : 2250-3021}

Vol. 2 Issue 2, Feb.2012, pp.310-314

administrators must fire up strategies to prioritize traffic, manage bandwidth and ensure application performance to these virtual desktops [8]. For any common systems, multiple $1 \mathrm{~Gb} / \mathrm{s}$ Ethernet LAN connections with additional multiple $8 \mathrm{~Gb} / \mathrm{s}$ Fiber Channel SAN storage connection is common practice.

3) Storage requirements: The storage infrastructure has very large impact on virtualized desktop instances. The primary deployment guideline should able to determine maximum storage capacity required as image storage, replication and backup user data generate enormous amount of data which needs to be stored efficiently. Client hosted desktop virtualization where image is stored and maintained at end user machine significantly reduces online data storage devices needed because it can be only used as dedicated offline backups [9].

\section{APPLICATIONS OF DESKTOP VIRTUALIZATION}

A Simple use of desktop virtualization involves remote administration where the controlling computer have more or less control on the client machine like local machine except those activities may be almost unnoticeable to the client machine like happens in using $\mathrm{X}$-session of GNU/Linux machine on remote machine. Here are five major approaches to desktop virtualization approaches and the types of situations they might be appropriate [10]

1) Remote Hosted Desktop: What most people think of when they think "terminal services" A server runs one image of an operating system or application and many clients $\log$ in to it using connection broker software that is the only part of the software hosted on the client machine. Client machines operate only to show an image on the monitor of the application that user is sharing, and to transmit keyboard and mouse input back and forth. The advantage of this approach is low cost and high degree control over data and applications and disadvantage of this approach is performance depends upon the quality of network connection and most peripherals or move data back and forth using thumb drives does not when disconnected. Citrix XenDesktop, Wyse ThinOS, Microsoft Remote desktop uses such type of approach.
2) Remote Virtual Application: It is used when many everyday Web applications delivers content which can be accessed by just browser and standard Web protocols (HTTP, HTTPS, SSL etc.) to create secure connection for transmission of data and graphics[11]. Advantages of this approach is that it doesn't require IT control the hardware and software environment of end user and disadvantage of this approach is that IT can't not control or have very less idea about end user environment, so it could affect performance. VMware View, VMware ThinApps use this type of approach.

3) Remote Hosted Dedicated Virtual Desktops: In this approach Increase users' capacity and reduce IT costs to protect the Web application or terminal services resources. The server does not allow many users to share the same application or instance of the operating system, and it only allows the user to access to a complete operating system or a set of applications in a virtual machine. This virtual machine can run on a server, or with other virtual machines dedicated to share resources, or own a blade PC. Advantage of this approach is it can run applications that balk at running in shares mode; isolates activity of each user to prevent resource constraints. Disadvantage of this is it uses far more bandwidth than shared desktops and more hardware on the server. Microsoft VDI suite, VMware View Manager uses this type of approach.

4) Local Virtual Applications: Consider java applets, they are downloaded from server to the client machine and run locally and usage local memory and processing power but still they run in some sandbox with limited interaction to host OS, it is example of local virtual application. Advantages of this approach are it gives better performance than remotely hosted application and consumes less bandwidth and also capable of running offline. Wyse TCX, Microsoft Application Virtualization uses this approach.

5) Local Virtual Operating System: There are two types of Local Virtual O.S. In first one a client side hypervisor can create a virtual machine within a laptop or desktop computer, which can function as completely standalone unit that keeps itself separate from hardware and software on the client machine outside of the virtualized desktop instance. In second type a 


\section{Pranit Patil, Shakti Shekar/ IOSR Journal of Engineering (IOSRJEN) \\ www.iosrjen.org \\ ISSN : 2250-3021}

Vol. 2 Issue 2, Feb.2012, pp.310-314

hypervisor runs on the machine's BIOS, allowing the user to run multiple virtualized instances with no 'host OS' at all. Advantages of this approach is the multiple OSes can run on single system with no concern of host OS. Disadvantage of this approach it can conflict resources if not properly managed. Citrix Dazzle and Receiver and Microsoft VDI suite are example of this approach.

\section{CONCLUSION}

The various advantageous of desktop virtualization like mobile computing, security, easier management and lower cost decides whether it will get support and enough research from industry. While all of the large vendors have offerings in the Desktop Virtualization space, the definition of the space is sufficiently broad.

Enterprises should carefully analyze the requirements for their various groups of users to understand what combinations of technologies fit each group best. Centralized VDI is a good fit for task oriented workers who all need the same set of applications delivered with absolute consistency. Client side hypervisors are a good solution when IT needs to completely lock down an environment, when users have the power to insist on their own flexibility, and when the users are sophisticated enough to be able to switch between two different operating systems and not get confused. Application and workspace isolation solutions lie in the middle of these two extremes offering combinations of both sets of benefits - and have the potential to appeal to the broadest set of users and administrators.

\section{REFERENCES}

1) http://www.itworld.com/virtualization/212245 /desktop-virtualization-smackdownparallels-vs-vmware-vs-virtualbox

2) http://www.desktopvirtualizationguide.org/

3) http://bit.ly/wWRefx

4)http://searchvirtualdesktop.techtarget.com/defi nition/desktop-virtualization

5) http://www.desktopvirtualizationguide.org/

6) http://en.wikipedia.org/wiki /Desktop_virtualization\#VDI_Modes_of_Op eration

7) http://www.cio.com/article/487109/Hosted Virtual_Desktop_Market_to_Cross_65_Billi on_in_2013/

8) http://searchnetworking.techtarget.com/tip /Desktop-virtualization-networkrequirements

9) A Smarter Approach to Desktop Virtualization The Virtualization Practice by Simon Bramfitt

10) http://www.cio.com/article/504348/Desktop_ Virtualization_5_Most_Popular_Flavors_Ex plain

11) Development and Application of Desktop Virtualization Technology by Li Yan 